\title{
Cardiovascular risk and endothelial function in people living with HIV/AIDS: design of the multi-site, longitudinal EndoAfrica study in the Western Cape Province of South Africa
}

Hans Strijdom ${ }^{1 *}$ (D), Patrick De Boever ${ }^{2,3}$, Gerhard Walzl ${ }^{4}$, M. Faadiel Essop ${ }^{5}$, Tim S. Nawrot $^{3,6}$, Ingrid Webster $^{1}$, Corli Westcott ${ }^{1}$, Nyiko Mashele ${ }^{1}$, Frans Everson ${ }^{1}$, Stephanus T. Malherbe ${ }^{4}$, Kim Stanley ${ }^{4}$, Harald H. Kessler ${ }^{7}$, Evelyn Stelzl ${ }^{\top}$ and Nandu Goswami ${ }^{8}$

\begin{abstract}
Background: There is growing evidence of an interaction between HIV-infection, anti-retroviral therapy (ART) and cardiovascular diseases (CVD). Epidemiological studies in Europe and North America have been observing a shift towards an increased incidence of coronary heart disease and acute myocardial infarctions in HIV-infected populations compared to the general population even after adjusting for traditional cardiovascular risk factors. Despite South Africa (and sub-Saharan Africa, SSA) being regarded as the epicentre of the global HIV epidemic, very little is known about the prevalence of cardiovascular risk factors and precursors of vascular disease in HIV-infected populations in this region. The knowledge gap is further widened by the paucity of data from prospective studies. We present the rationale, objectives and key methodological features of the EndoAfrica study, which aims to determine whether HIVinfection and ART are associated with altered cardiovascular risk and changes in vascular endothelial structure and function in adults living in the Western Cape Province of South Africa.
\end{abstract}

Methods: In this longitudinal study, comprehensive cardiovascular assessments of HIV-negative and HIV-positive (with and without ART) study participants are performed by clinical and biochemical screening for traditional cardiovascular risk factors and biomarkers of CVD. Vascular and endothelial function is determined by brachial artery flow-mediated dilatation (FMD), carotid-intima-thickness (IMT) measurements and quantitative retinal blood vessel analyses, complemented by vascular endothelial biomarker assays. Finally, we aim to statistically determine whether HIVinfection and/or ART are associated with increased cardiovascular risk and vascular endothelial dysfunction, and determine whether there is progression/regression in these endpoints 18 months after the baseline assessments.

Discussion: The EndoAfrica study provides a unique opportunity to recruit a cohort of HIV-infected patients and HIVnegative controls who will be comprehensively and longitudinally assessed for cardiovascular risk and disease profile with vascular endothelial function as a potentially important intermediate cardiovascular phenotype. To our knowledge, it is the first time that such a systematic study has been established in the context of SSA and South Africa.

Keywords: HIV, Antiretroviral therapy, Cardiovascular risk factors, Vascular endothelial function, South Africa

\footnotetext{
*Correspondence: jgstr@sun.ac.za

'Division of Medical Physiology, Faculty of Medicine and Health Sciences,

Stellenbosch University, PO Box 241, Cape Town 8000, South Africa

Full list of author information is available at the end of the article
} 


\section{Background}

There is growing evidence of an interplay between HIVinfection, anti-retroviral therapy (ART) and cardiovascular diseases (CVD) [1]. The trends with regards to the nature of HIV-related CVD have evolved since the introduction of highly active ART (HAART). HIV-associated cardiomyopathy was one of the first CVD conditions described in HIV-infected study populations [2-5], with systolic dysfunction particularly prevalent in the pre-HAART era. Since the introduction of HAART, epidemiological studies in Europe and North America have been observing a shift towards an increased incidence of coronary heart disease in HIV-infected populations compared to the general population [1, 6-8]. For example, a large prospective cohort study in the USA with $\sim 82,000$ participants showed that HIV-infected participants had a $50 \%$ increased risk of acute myocardial infarction even after adjusting for traditional cardiovascular risk factors [9].

Sub-Saharan Africa (SSA) is home to approximately 26million (70\%) of the world's HIV-infected population [10]. As a result, SSA has witnessed the roll-out of massive ART programmes in recent years. For example, South Africa has the world's largest Government-sponsored programme that reached approximately 3.1-million people in 2015 [11]. In addition to the HIV/AIDS epidemic, many countries in SSA are subjected to a rapidly expanding non-communicable diseases epidemic, characterised by, among others, a high prevalence of traditional cardiovascular risk factors and disease [12]. In fact, it has been reported that $80 \%$ of all cardiovascular-related deaths globally occur in low- to middle income countries [13]. In South Africa, the growing prevalence of traditional cardiovascular risk factors such as hypertension, obesity and diabetes mellitus has been described as a "time bomb" [14], and the multiple burden of communicable and noncommunicable diseases a "collision of epidemics" [15]. Surprisingly, the impact of high HIV-infection rates and HAART on the increasing prevalence of CVD in SSA has received relatively little research attention and the knowledge gap is further widened by the paucity of data from longitudinal studies.

Based on the limited epidemiological data available, pulmonary hypertension, cardiomyopathy and tuberculous pericarditis are reported to be the most common cardiovascular-related pathologies associated with HIVinfection in SSA populations $[12,16]$. The incidence of coronary artery disease in SSA is relatively low, not only in the general population [17], but seemingly also in people affected by HIV-infection [18]. However, there is reason to believe that the current predominantly "preHAART" cardiovascular disease profile of sub-Saharan Africans living with HIV/AIDS may change. Taking into account the increase in coronary artery disease observed in HIV-infected populations from Europe and USA since the introduction of HAART, it is conceivable that the full cardiovascular health impact of the HAART rollout programmes initiated in SSA in recent years has not yet been felt [12]. In this regard, data are emerging from countries such as Kenya and South Africa showing a higher incidence of traditional (predominantly pro-atherogenic) cardiovascular risk factors, such as hypertension, obesity and dyslipidaemia in HIVinfected participants [19-21].

Vascular endothelial activation and endothelial dysfunction are now recognized as early markers of HIV-related CVD [1, 22, 23], and recent data from South Africa confirmed that HIV-infection was associated with increased inflammation-induced endothelial injury, endothelial activation, and endothelial dysfunction [24, 25]. Vascular endothelial changes occur early in the cardiovascular disease process, and endothelial dysfunction is widely recognized as a marker of the net effects of cardiovascular risk factors as well as future cardiovascular events [26]. Hence, the inclusion of vascular endothelial function assessment as a surrogate marker of CVD in clinical research in SSA populations has recently been advocated $[27,28]$.

\section{Problem statement}

The HIV/AIDS epidemic in SSA appears to be on a collision course with the rapidly increasing burden of cardiovascular risk factors and disease. However, the scope and nature of the cardiovascular risk and disease profile, including early vascular and endothelial changes, of HIV-infected populations in SSA remains unclear. Hence, the need for epidemiological studies to address this knowledge gap is great. Studies should screen for traditional endpoints of cardiovascular risk in HIV/ AIDS-exposed populations, and also incorporate endpoints that provide early (preclinical) and predictive information on cardiovascular risk and future events, such as vascular endothelial function measurements.

The EndoAfrica study was established to provide a comprehensive and repeated assessment of the cardiovascular risk and disease profile in South African adults living with HIV/AIDS. The study includes clinical screening and extensive biomarker analysis of vascular endothelial function. To our knowledge, it is the first time that such a systematic study has been established in the context of SSA and South Africa.

\section{Methods/design}

\section{Aim and objectives}

The overarching aim of the EndoAfrica study is to determine whether HIV-infection and ART are associated with altered cardiovascular risk and changes in vascular endothelial structure and function in adults living in the Western Cape Province of South Africa. The following objectives have been defined: 
1. To perform comprehensive cardiovascular assessments of HIV-negative and HIV-positive (with and without ART) study participants by clinical and biochemical screening for traditional cardiovascular risk factors (overweight/obesity, smoking, hypertension, diabetes mellitus, dyslipidaemia) and biomarkers of CVD;

2. To perform assessments of vascular and endothelial function by ultrasound-based flow-mediated dilatation (FMD) measurements of the brachial artery, carotid-intima-thickness (IMT) measurements, quantitative retinal blood vessel analysis, and vascular endothelial biomarker analyses;

3. To determine statistically whether HIV-infection and/or ART are associated with increased cardiovascular risk and vascular endothelial dysfunction, and determine whether there is progression/regression in these endpoints 18 months after the baseline assessments.

\section{Study design and setting}

EndoAfrica uses a repeated measures design, and study participants undergo baseline and 18 months' follow-up assessments. Assessments include a personal interview and completion of a comprehensive health questionnaire, standard anthropometric measurements, a cardiovascular system-focused physical examination, blood and urine collection for biochemical and biomarker analyses, ultrasound-based vascular and endothelial assessments (FMD and carotid IMT), and fundus imaging for retinal blood vessel analysis. The study allows the investigators to monitor the progression or regression of the major study endpoints in participants over a 18-month period. EndoAfrica, which commenced officially in April 2015, has received ethics approval from the relevant body at Stellenbosch University (see later) and is conducted in Cape Town and Worcester in the Western Cape Province of South Africa.

\section{Participant recruitment and study groups}

The study population mainly consists of patients visiting primary health care clinics. Participants are recruited by qualified and experienced research nurses at community health care centres and/or HIV clinics in Cape Town (Elsies River, Adriaanse, Fisantekraal, Uitsig, Durbanville and Ravensmead), and the local community health centre in Worcester based on pre-determined inclusion and exclusion criteria. For inclusion into the study, participants must be 18 years or older, and female participants must not be pregnant or less than 3 months post-partum. Participants will be excluded from the study if they are younger than 18 years, pregnant, or less than 3 months post-partum. Participants who qualify for inclusion are invited to take part in the study and requested to sign an informed consent form.

Once enrolled, participants with unknown HIV status are counselled for HIV testing and screened for HIVinfection by performing a rapid HIV test (SD Bioline HIV 1/2 3.0 immunochromatographic test kit; Standard Diagnostics, Republic of Korea). If the test result is negative, the participant is assigned to the HIV-negative control group. Participants with confirmed HIV-infection are assigned to the HIV-positive group, which is further divided into two sub-groups based on the ART status of participants. Those participants not receiving ART are assigned to the "HIV-positive ART naïve" sub-group. Current South African ART guidelines recommend that all newly diagnosed HIV-infected patients be placed on ART regardless of their CD4 count. In view of this, one has to realistically assume that the majority of participants in this sub-group will likely commence with ART soon after recruitment. This provides a unique opportunity to assess cardiovascular risk and early endothelial changes in both the untreated (baseline) and treated (18 months' follow-up) state in the same participants. Therefore, the effects of ART can be assessed in individuals who serve as their own untreated controls. The second sub-group consists of HIV-infected participants who have been on ART for at least four weeks ("HIVpositive on ART"). The CD4 count and viral load of the HIV-positive participants will be determined at baseline and 18 months' follow-up. All participants are required to fast from $22 \mathrm{~h} 00$ the night before the assessments. They are also asked to refrain from smoking, drinking coffee or doing exercise for 4-6 h prior to the study, and female participants should report the phase of their menstrual cycle. Figure 1 depicts the study design, inclusion/exclusion criteria and study groups.

\section{Assessments: clinical and laboratory investigations Health questionnaire, anthropometry, cardiovascular measurements, blood and urine chemistry}

General cardiovascular health information is obtained from questionnaires and personal interviews (including family and personal history of cardiovascular disease, medication, smoking, and alcohol consumption). In addition, participants are screened for cardiovascular risk factors by anthropometric measurements, heart rate and blood pressure measurements, and blood and urine chemistry analyses. The anthropometric assessments, viz. body-mass-index (BMI), waist circumference and waist-to-hip ratio, are performed according to international standards [29]. Heart rate and brachial systolic and diastolic blood pressure of the left arm are measured at three different occasions, 25 min apart, with an Omron M6 automatic digital blood pressure monitor (Omron Healthcare, Kyoto, Japan). Blood and early morning mid-stream urine samples are obtained 


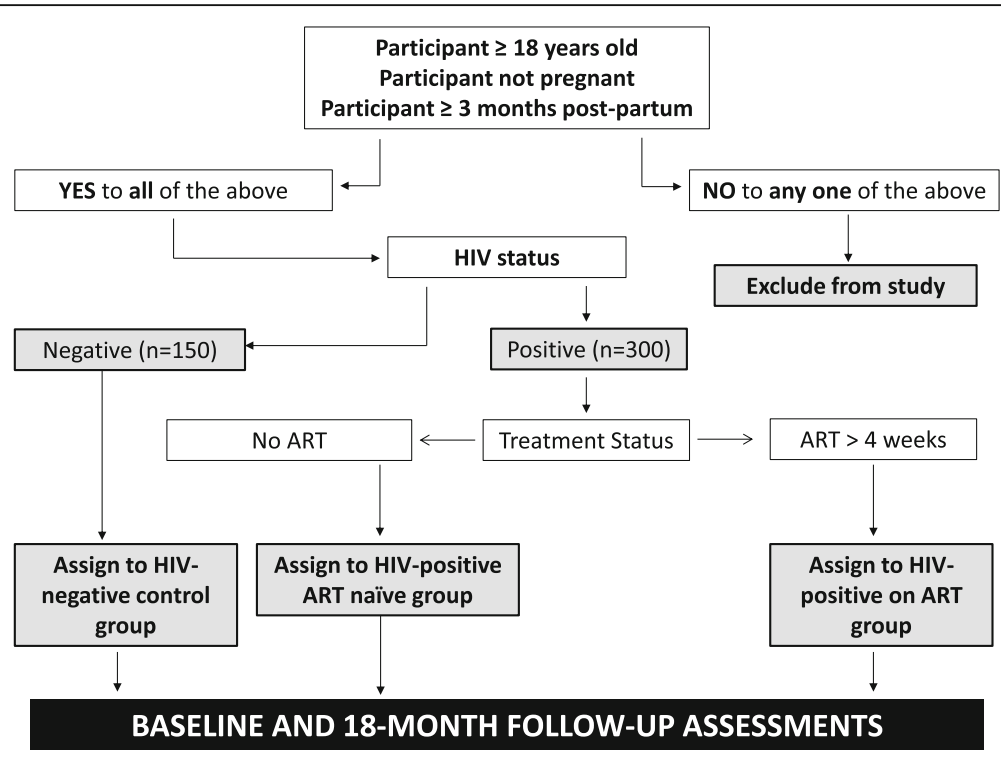

Fig. 1 Recruitment protocol and study groups

by the research nurse from each participant. Samples are transported to the National Health Laboratory Service (NHLS) laboratory in the Tygerberg Academic Hospital, adjacent to the Faculty of Medicine and Health Sciences of Stellenbosch University. Here, the following markers of cardiovascular risk are measured: (i) Lipids (total cholesterol, LDL-cholesterol, HDL-cholesterol and triglycerides), (ii) glucose, (iii) glycated haemoglobin (HbA1c), (iv) creatinine, (v) gamma-glutamyl transferase (GGT), (vi) highly sensitive C-reactive protein (hs-CRP), (vii) haemoglobin, and (viii) urine albumin : creatinine ratio. The measurements are performed according to NHLS approved standard analytical protocols. Collectively, results from the above interviews and investigations allow the researchers to screen for the following cardiovascular risk factors: smoking, alcohol consumption, overweight/obesity, hypertension, diabetes mellitus, dyslipidaemia, renal impairment, inflammation and liver disease.

\section{Flow mediated dilation (FMD)}

FMD is regarded as the gold standard of noninvasive vascular endothelial function assessment in the research setting [26, 30]. Several studies have been performed in which FMD measurement of vascular endothelial function was validated in terms of prognostic ability as well as successful disease progression/regression monitoring in clinical trials [30]. FMD has also previously been shown to be a marker of effect in populations living with HIV/AIDS [31, 32].

The FMD procedure used in the EndoAfrica study is based on a previously described protocol [30], and performed by research assistants who have been trained by specialists in the field. According to the protocol, the participant rests in the supine position for 10 min prior to the procedure. A longitudinal image of the right brachial artery is obtained in B-mode with an Esaote MyLab ${ }^{\mathrm{TM}}$ Five ultrasound and $12 \mathrm{MHz}$ linear probe (Esaote, Italy). The probe is stabilised on the upper-arm using a single axis precision probe holder from SMT Medical (Wuerzburg, Germany) that allows for micro-metric adjustments. Doppler mode is used to visualise and locate the brachial artery with pulse repetition frequency (PRF) set at $6.7 \mathrm{kHz}$. Once the artery is properly visualised, depth is increased to $3 \mathrm{~cm}$ before switching to pulse wave mode. In pulse wave mode, the angle of insonation is set to $+60^{\circ}$. Brachial artery diameter and shear rate measures are recorded continuously with FMD Studio and Cardiovascular Suite version 2.8.1 software (Quipu, Italy), which makes use of automatic edge detection technology. The protocol starts with 1 min baseline recording at rest. Following this, a blood pressure cuff around the fore-arm is inflated to $50 \mathrm{mmHg}$ above the systolic blood pressure value and remains inflated for $5 \mathrm{~min}$, this is called the ischaemic phase. After $5 \mathrm{~min}$, the cuff is deflated resulting in hyperaemia and increased shear rate inducing endothelium-dependent dilatation of the brachial artery, followed by the recovery phase (total recording time: $3 \mathrm{~min}$ ). At the end of the procedure, the software calculates baseline artery diameter, maximum artery diameter, recovery artery diameter, FMD \% (difference between maximum and baseline diameter expressed as \%), baseline shear rate and maximum shear rate.

\section{Carotid intima-media thickness (IMT)}

Carotid IMT is an important biomarker of arterial wall thickening and subclinical and clinical atherosclerosis [33, 34]. Increases in IMT are associated with both 
prevalent and incident cardiovascular morbidity and mortality, including coronary heart disease [35]. IMT measurements will follow protocols and guidelines as described previously [36]. The participants are in the supine position with the head tilted $30^{\circ}$ to the left for assessment of the right carotid artery, and $30^{\circ}$ to the right for assessment of the left carotid artery with the Esaote MyLab $^{\mathrm{TM}}$ Five ultrasound and $12 \mathrm{MHz}$ linear probe (Esaote, Italy). Once the carotid artery bifurcation is visualised manually in b-mode, a $10 \mathrm{~mm}$ straight arterial segment is selected in the common carotid artery proximal to the bifurcation bulb. The segment must be free of atherosclerotic plaque and the lumen-intima and media-adventitia interfaces must be clearly defined. Dedicated Quality Intima-Media Thickness (QIMT) software is used for real-time radio frequency detection of the IMT, which automatically measures the intima-media thickness. The software additionally calculates the median \pm standard deviation IMT values during several cardiac cycles [37].

\section{Retinal vessel analyses}

Retinal image analysis is an unobtrusive procedure for visualizing the microcirculation. The microcirculation consists of blood vessels less than $\sim 150 \mu \mathrm{m}$ and includes smallest resistance arteries, arterioles, capillaries, and venules. These vessels make up a large part of the circulatory system and play an important role in maintaining cardiovascular health. There is substantial evidence in favour of a correlation between retinal vascular changes and coronary heart disease $[38,39]$. The ratio between the diameter of retinal arteries and retinal veins $(\mathrm{A} / \mathrm{V})$ has been shown to be a sensitive proxy to reflect hypertension and atherosclerosis [40]. A narrowing of the arteries and widening of the veins, leading to a decreased $\mathrm{A} / \mathrm{V}$ ratio, corroborates with risk of stroke and myocardial infarction [41]. Quantitative features extracted from retinal images have been proven useful for identifying microvascular changes with predictive power in the field of cardiovascular disease development [42].

Fundus images are captured with a non-mydriatic digital retinal camera (Canon CR-2 camera, Canon Europa NV, The Netherlands). The retinal vessel dimensions, vascular tree and microvascular state are analysed with semi-automated IFLEXIS software developed by VITO (Belgium). Analysis of retinal images are undertaken by a trained grader, masked to participant characteristics. The grader performs the vessel measurements on the optic disc-centred image of both the right and left eye; however, the data of the right eye are used for most participants and left eye data only when the right eye images are ungradable. The software allows calculating vessels widths and pattern features. For the calculation of vessel width, the largest six arterioles and venules coursing through a zone between 0.5 and 1 disc diameter from the optic disc margin are measured. Estimates are summarized as central arteriolar equivalent (CRAE) and central retinal venular equivalent (CRVE), representing the average diameter of arterioles and venules of the eye, respectively [43]. For the calculation of pattern features such as tortuosity, fractal analysis, and lacunarity, all blood vessels coursing through a zone of $2 x$ disc diameter from the optic disc margin are measured. An overview of the different retinal features that are calculated are also reported in a recent study by Prabhakar and colleagues [44].

\section{Serum biomarker analyses}

An array of serum biomarkers of vascular endothelial dysfunction will be measured to complement the clinical measurements. The following biomarkers have previously been shown to be associated with vascular endothelial dysfunction, and will be measured in this study: vascular cellular adhesion molecule-1 (VCAM-1), intercellular adhesion molecule-1 (ICAM-1), e-selectin, p-selectin, platelet activating inhibitor-1 (PAI-1), tumor necrosis factor-alpha (TNF- $\alpha$ ), vascular endothelial growth factor (VEGF) and von Willebrand Factor [22]. These markers will be measured by Luminex technology (Luminex BioPlex 200 system) in the Division of Molecular Biology and Human Genetics, Faculty of Medicine and Health Sciences, Stellenbosch University. The Luminex cytokine/ chemokine/protein assays are sandwich immunoassays that are performed in 96-well filter plates, similar to sandwich ELISA assays, and can analyse up to 100 different host markers in each well.

Figure 2 shows the baseline and 18 months' follow-up assessments.

\section{Data collection, management and statistical analysis}

All data generated from the health questionnaires, clinical assessments and biochemical investigations will be collected and managed using REDCap electronic data capture tools hosted at the Department of Biomedical Sciences,

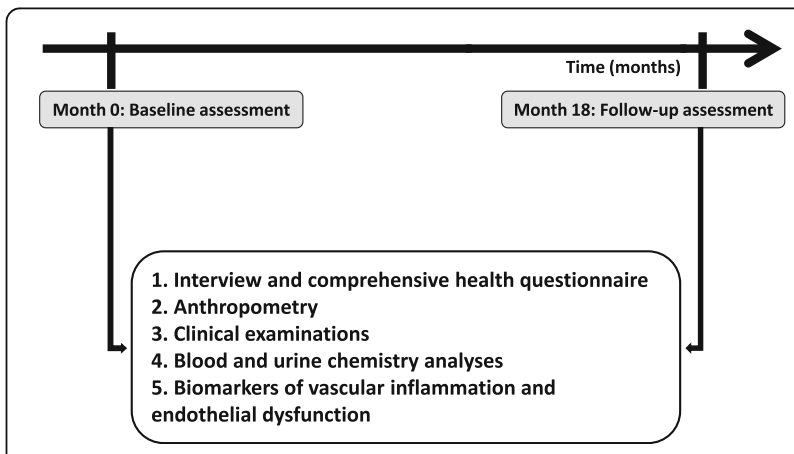

Fig. 2 Clinical and biochemical assessments of study participants at baseline and 18 months' follow-up 
Stellenbosch University [45]. REDCap (Research Electronic Data Capture) is a secure, web-based application designed to support data capture for research studies.

Data are statistically analyzed in conjunction with the Biostatistics Unit in the Centre for Evidence-based Health Care, Stellenbosch University. Descriptive statistical models (ANOVA, Chi Square) will be used for inter-group comparisons, and associations between independent and dependent variables will be tested by multivariate regression analysis models, adjusting for age, gender, ethnicity, smoking, alcohol consumption, medication and BMI. For longitudinal changes within the same individual, a repeated measures model approach will be employed.

A literature search was undertaken with the assistance of the Biostatistics Unit (Faculty of Medicine and Health Sciences, Stellenbosch University), and according to Donald et al. [46], a case sample size of between $n=100-400$ over a 3 months' follow-up period was sufficiently powerful to secure endothelial function measurement reproducibility and statistical significance (1-2\% effect size; $80 \%$ statistical power and 5\% significance). In our own pilot studies performed in 2015 and early 2016, several cardiovascular risk factors (BMI, HDL-cholesterol, blood pressure) were significantly different between HIV-negative control and HIVpositive participants, and HIV-infection was associated with increased triglyceride levels and highly sensitive CRP values (HIV-positive sample size: $n=110$ ). We are therefore confident that the proposed sample sizes will be sufficient to secure sufficient statistical power.

At the time of writing, EndoAfrica has recruited a total of 231 participants, of whom 60 are HIV-negative control and 171 are HIV-infected (ART naïve: $n=38$ ). The characteristics of the cohort at this stage show a mean age of $39.23 \pm 9.98$ years, predominantly female participants $(74.8 \%)$ with a high smoking prevalence $(60.2 \%)$. The study is on-going, and provisional completion date is December 2018.

See Fig. 3 for a flow chart showing an overview of the study and operating procedures.

\section{Discussion}

The EndoAfrica study will generate novel data in the South African (and SSA) context. We are not aware of any previous longitudinal studies in sub-Saharan Africa undertaken to comprehensively assess cardiovascular

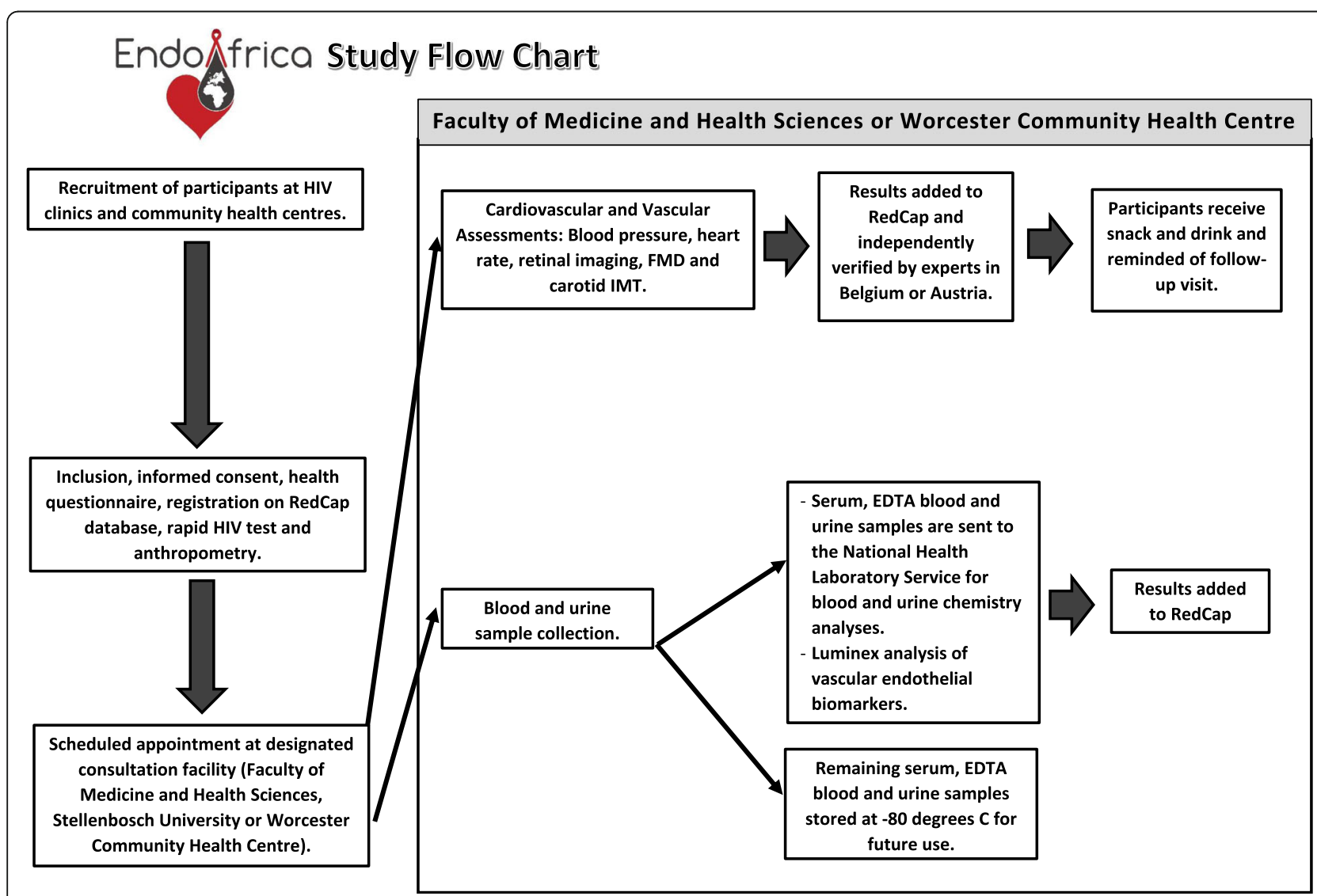

Fig. 3 Study overview and operating procedures. FMHS: Faculty of Medicine and Health Sciences; FMD: flow-mediated dilatation; IMT: intima-media thickness; NHLS: National Health Laboratory Service 
risk and vascular endothelial changes in adults living with and without HIV/AIDS. The repeated measures design of EndoAfrica will provide valuable insights into the progression or regression of markers of cardiovascular risk and disease in HIV-infected subjects. This information can help to develop guidelines for the management of cardiovascular health in patients with HIV-infection in the local health care system. The findings of the EndoAfrica study will furthermore contribute to the current global knowledge base on HIV and cardiovascular disease, which is largely devoid of data from SSA.

The vascular and endothelial endpoints of the EndoAfrica study will not only generate novel research data in the South African context, but also have the potential to predict future cardiovascular events. With a few exceptions, very little data are available on endothelial dysfunction in adult SSA populations in general, and in HIV-infected populations in particular. The fact that three independent, non-invasive imaging techniques, complemented by the measurement of a battery of serum biomarkers will be employed, can be regarded as a strength of the study.

\section{Possible limitations}

The ethnic composition of the Western Cape Province of South Africa, and hence of our study cohort, may limit the possibility to extrapolate the findings to the rest of the population. The majority of the population in Cape Town and Worcester is of mixed ancestry, whilst the majority of HIV-infected South Africans are ethnic black Africans. The researchers will endeavour to recruit as many black study participants as possible, and steps to incorporate recruitment sites in other provinces where more black participants can be included are currently undertaken. In addition, the follow-up period of 18 months may be too short to sufficiently determine the progression of cardiovascular risk and vascular changes. However, there have been previous prospective studies employing similar or shorter follow-up periods that could demonstrate disease progression or regression (e.g. increased carotid IMT progression in HIV-infected participants after 12 months [47], and regression of endothelial dysfunction biomarkers after median 7.2 months ART [48]). The 18-month follow-up period was chosen in view of the total project duration of 3 years. The EndoAfrica research team is now building capacity and developing sustainable collaboration with the aim to extend the follow-up period of the longitudinal cohort beyond the lifetime of the initial project.

Recently, the South African Government announced that all HIV-infected patients will immediately commence with ART compared to the previous guidelines of initiating ART at a CD4 count cut-off of 500 cells/ $\mu \mathrm{L}$. This may limit our ability to recruit ART naïve participants. Finally, the researchers need to take cognizance of the fact that the FMD and IMT procedures have certain limitations in terms of operator dependency and inter-operator variability. Ensuring that the same vascular regions are assessed in each participant at the 18 months' follow-up visit is also a potential challenge. In order to address this as far as possible, we will limit the number of operators in the team, subject them to intensive and expert training, and employ rigorous verification and quality control measures by independent experts in this field.

\section{Conclusion}

EndoAfrica is an important and relevant study in the context of cardiovascular risk and vascular endothelial dysfunction in HIV-infection. South Africa (and subSaharan Africa) is the epicentre of the global HIV epidemic, yet very little is known about the prevalence of cardiovascular risk factors and early vascular disease in HIV-infected populations in this region. The EndoAfrica study addresses this knowledge gap.

\begin{abstract}
Acknowledgements
Not applicable.

Funding

The EndoAfrica study is receiving funding from the ERAfrica program of the EU $7^{\text {th }}$ Framework Program. Funding is disbursed via the Department of Science and Technology in South Africa (contract number DST/CON 0077/ 2014), the Belgian Science Policy in Belgium (contract number BL/67/ eranet03), and Österreichische Agentur für internationale Mobilität und Kooperation in Bildung, Wissenschaft und Forschung, OeAD GmbH (ÖAD) in Austria. IW, CW and NM are supported by the South Africa National Research Foundation (NRF) post-doctoral scholarship program and FE by the NRF doctoral scholarship program.
\end{abstract}

\section{Authors' contributions}

HS, PDB, TSN and NG wrote the original grant proposal, and HS is the project coordinator. The manuscript outline and draft was developed by HS, and refined and edited by all the authors. The final manuscript was reviewed and approved by all the authors.

\section{Competing interests}

The authors declare that they have no competing interests.

\section{Ethics approval and consent to participate}

The EndoAfrica study has received ethics approval from the Health Research Ethics Committee (HREC) of Stellenbosch University (HREC reference numbers: N13/05/064 and S16/07/114), and is conducted according to the ethical guidelines and principles of the international Declaration of Helsinki, South African Guidelines for Good Clinical Practice and the Medical Research Council of South Africa Ethical Guidelines for Research.

\footnotetext{
Author details

${ }^{1}$ Division of Medical Physiology, Faculty of Medicine and Health Sciences, Stellenbosch University, PO Box 241, Cape Town 8000, South Africa. ¿Environmental Risk and Health Unit, Flemish Institute for Technological Research (VITO), Boeretang 200, 2400 Mol, Belgium. ${ }^{3}$ Centre for

Environmental Studies, Hasselt University, Agoralaan, 3590 Diepenbeek, Belgium. ${ }^{4}$ Division of Molecular Biology and Human Genetics, DST/NRF Centre of Excellence for Biomedical Tuberculosis Research and SAMRC Centre for Tuberculosis Research, Faculty of Medicine and Health Sciences, Stellenbosch University, PO Box 241, Cape Town 8000, South Africa. ${ }^{5}$ Cardio-Metabolic Research Group (CMRG), Department of Physiological Sciences, Faculty of Science, Stellenbosch University, Stellenbosch 7600,
} 
South Africa. ${ }^{6}$ Department of Public Health and Primary Care, Leuven University, Kapucijnenvoer 35, 3000 Leuven, Belgium. 'Institute of Hygiene, Microbiology and Environmental Medicine, Medical University of Graz, Universitätsplatz 4, 8010 Graz, Austria. ${ }^{8}$ Gravitational Physiology and Medicine Research Unit, Institute of Physiology, Medical University of Graz, Harrachgasse 21N, 8010 Graz, Austria.

\section{Received: 7 December 2016 Accepted: 22 December 2016}

Published online: 07 January 2017

\section{References}

1. Boccara F, Lang S, Meuleman C, Ederhy S, Mary-Krause M, Costagliola D, et al. HIV and coronary heart disease: time for a better understanding. J Am Coll Cardiol. 2013;61(5):511-23.

2. Levy WS, Simon GL, Rios JC, Ross AM. Prevalence of cardiac abnormalities in human immunodeficiency virus infection. Am J Cardiol. 1989;63:86-9.

3. Herskowitz A, Vlahov D, Willoughby S, Chaisson RE, Schulman SP, Neumann DA, et al. Prevalence and incidence of left ventricular dysfunction in patients with human immunodeficiency virus infection. Am J Cardiol. 1993; 71:955-8.

4. De Castro S, Migliau G, Silvestri A, D'Amati G, Giannantoni P, Cartoni D, et al. Heart involvement in AIDS: a prospective study during various stages of the disease. Eur Heart J. 1992;13:1452-9.

5. De Castro S, d'Amati G, Gallo P, Cartoni D, Santopadre P, Vullo V, et al. Frequency of development of acute global left ventricular dysfunction in human immunodeficiency virus infection. J Am Coll Cardiol. 1994;24:1018-24

6. Currier JS, Lundgren JD, Carr A, Klein D, Sabin CA, Sax PE, et al. Epidemiological evidence for cardiovascular disease in HIV-infected patients and relationship to highly active antiretroviral therapy. Circulation. 2008;118:e29-35.

7. Lang S, Mary-Krause M, Cotte L, Gilquin J, Partisani M, Simon A, et al. Increased risk of myocardial infarction in HIV-infected patients in France, relative to the general population. AIDS. 2010;24(8):1228-30.

8. Friis-Møller N, Sabin CA, Weber R, d'Arminio Monforte A, El-Sadr WM, Reiss $P$, et al. Combination antiretroviral therapy and the risk of myocardial infarction. N Engl J Med. 2003;349(21):1993-2003.

9. Freiberg MS, Chang CC, Kuller LH, Skanderson M, Lowy E, Kraemer KL, et al. HIV infection and the risk of acute myocardial infarction. JAMA Intern Med. 2013;173(8):614-22.

10. Joint United Nations Programme on HIV/AIDS (UNAIDS) Factsheet 14 July 2015. MDG 6: 15 years, 15 lessons of hope from the AIDS response. http:// www.unaids.org/en/resources/documents/2015/MDG6_15years15lessonsfromtheAIDSresponse. Accessed 7 Aug 2016.

11. Department of Health, South Africa HIV \& AIDS 2014/2015 Data Factsheet. https://www.health-e.org.za/wp-content/uploads/2015/06/2014-15-HIV-DataFact-Sheet-01-June-2015.pdf. Accessed 7 Aug 2016.

12. Mayosi BM, Flisher AJ, Lalloo UG, Sitas F, Tollman SM, Bradshaw D. The burden of non-communicable diseases in South Africa. Lancet. 2009; 374:934-47

13. Yusuf S, Rangarajan S, Teo K, Islam W, Li L, Liu J, et al. Cardiovascular risk and events in 17 low-, middle-, and high-income countries. N Engl J Med. 2014;371:818-27.

14. Tibazarwa K, Ntyintyane L, Sliwa K, Gerntholz T, Carrington M, Wilkinson $D$, et al. A time bomb of cardiovascular risk factors in South Africa: results from the Heart of Soweto Study "Heart Awareness Days". Int J Cardiol. 2009;132:233-9.

15. Mayosi BM, Lawn JE, van Niekerk A, Bradshaw D, Karim SSA, Coovadia HM for The Lancet South Africa team. Health in South Africa: changes and challenges since 2009. Lancet. 2012;380:2029-43.

16. Syed FF, Sani MU. Recent advances in HIV-associated cardiovascular diseases in Africa. Heart. 2013;99:1146-53.

17. Sliwa K, Acquah L, Gersh BJ, Mocumbi AO. Impact of Socioeconomic Status, Ethnicity, and Urbanization on Risk Factor Profiles of Cardiovascular Disease in Africa. Circulation. 2016;133:1199-208.

18. Sliwa K, Carrington MJ, Becker A, Thienemann F, Ntsekhe M, Stewart S. Contribution of the human immunodeficiency virus/acquired immunodeficiency syndrome epidemic to de novo presentations of heart disease in the Heart of Soweto Study cohort. Eur Heart J. 2012:33(7):866-74.

19. Bloomfield GS, Hogan JW, Keter A, Sang E, Carter EJ, et al. Hypertension and Obesity as Cardiovascular Risk Factors among HIV Seropositive Patients in Western Kenya. PLoS ONE. 2011;6(7):e22288.
20. Julius H, Basu D, Ricci E, Wing J, Basu JK, Pocaterra D, Velazquez EJ, et al. The burden of metabolic diseases amongst HIV positive patients on HAART attending the Johannesburg Hospital. Curr HIV Res. 2011;9(4):247-52.

21. Temu TM, Kirui N, Wanjalla C, Ndungu AM, Kamano JH, Inui TS, et al. Cardiovascular health knowledge and preventive practices in people living with HIV in Kenya. BMC Infect Dis. 2015;15:421.

22. Graham SM, Mwilu R, Liles WC. Clinical utility of biomarkers of endothelial activation and coagulation for prognosis in HIV infection: a systematic review. Virulence. 2013;4(6):564-71.

23. Skowyra A, Zdziechowicz I, Mikuła T, Wiercinska-Drapało A. Endothelial dysfunction - An important factor in the progression of atherosclerosis in HIV-infected persons. HIV AIDS Rev. 2012;11:57-60.

24. Fourie CM, Schutte AE, Smith W, Kruger A, van Rooyen JM. Endothelial activation and cardiometabolic profiles of treated and never-treated HIV infected Africans. Atherosclerosis. 2015;240(1):154-60.

25. Fourie C, van Rooyen J, Pieters M, Conradie K, Hoekstra T, Schutte A. Is HIV-1 infection associated with endothelial dysfunction in a population of African ancestry in South Africa? Cardiovasc J Afr. 2011;22(3):134-40.

26. Deanfield JE, Halcox JP, Rabelink TJ. Endothelial function and dysfunction: testing and clinical relevance. Circulation. 2007;115:1285-95.

27. Sampson UK, Engelgau MM, Peprah EK, Mensah GA. Endothelial dysfunction: a unifying hypothesis for the burden of cardiovascular diseases in sub-Saharan Africa. Cardiovasc J Afr. 2015;26(2 Suppl 1):S56-60.

28. Strijdom $\mathrm{H}$. Endothelial dysfunction: are we ready to heed the vasculature's early-warning signal? Cardiovasc J Afr. 2012;23(4):184-5.

29. Stewart A, Marfell-Jones M. International standards for anthropometric assessment. 2011th ed. New Zealand: Lower Hunt; 2011.

30. Charakida M, Masi S, Lüscher TF, Kastelein JJ, Deanfield JE. Assessment of atherosclerosis: the role of flow-mediated dilatation. Eur Heart J. 2010;1(23): 2854-6.

31. Stein JH, Brown TT, Ribaudo HJ, Chen Y, Yan M, Lauer-Brodell E, McComsey GA, Dubé MP, Murphy RL, Hodis HN, Currier JS. Ultrasonographic measures of cardiovascular disease risk in antiretroviral treatment-naive individuals with HIV infection. AIDS. 2013:27(6):929-37.

32. Ho JE, Scherzer R, Hecht FM, Maka K, Selby V, Martin JN, Ganz P, Deeks SG, Hsue PY. The association of CD4+ T-cell counts and cardiovascular risk in treated HIV disease. AIDS. 2012;26(9):1115-20.

33. Bauer M, Caviezel S, Teynor A, Erbel R, Mahabadi AA, Schmidt-Trucksäss A Carotid intima-media thickness as a biomarker of subclinical atherosclerosis. Swiss Med Wkly. 2012;142:w13705.

34. de Groot E, Hovingh GK, Wiegman A, Duriez P, Smit AJ, Fruchart JC, et al. Measurement of arterial wall thickness as a surrogate marker for atherosclerosis. Circulation. 2004;109(23 Suppl 1):III33-8.

35. Plichart M, Celermajer DS, Zureik M, Helmer C, Jouven X, Ritchie K, et al. Carotid intima-media thickness in plaque-free site, carotid plaques and coronary heart disease risk prediction in older adults. The Three-City Study. Atherosclerosis. 2011:219(2):917-24.

36. Simova I. Intima-media thickness: appropriate evaluation and proper measurement, described. E-journal of the ESC Council for cardiology practice. 2015; 13(21). http://www.escardio.org/Journals/E-Journal-ofCardiology-Practice/Nolume-13/Intima-media-thickness-Appropriateevaluation-and-proper-measurement-described. Accessed 7 Aug 2016.

37. Ciccone MM, Scicchitano P, Zito A, Agati L, Gesualdo M, Mandolesi S, et al Correlation between coronary artery disease severity, left ventricular mass index and carotid intima media thickness, assessed by radio-frequency. Cardiovasc Ultrasound. 2011;9:32

38. Liew G, Wang JJ, Mitchell P, Wong TY. Retinal Vascular Imaging A New Tool in Microvascular Disease Research. Circ Cardiovasc Imaging. 2008;1:156-61.

39. McGeechan K, Liew G, Wong TY. Are retinal examinations useful in assessing cardiovascular risk? Am J Hypertens. 2008;21:847.

40. McClintic BR, McClintic Jl, Bisognano JD, Block RC. The relationship between retinal microvascular abnormalities and coronary heart disease: a review. Am J Med. 2010:123:e1-e374.

41. Hubbard LD, Brothers RJ, King WN, Clegg LX, Klein R, Cooper LS, et al. Methods for evaluation of retinal microvascular abnormalities associated with hypertension/sclerosis in the atherosclerosis risk in communities study. Ophthalmology. 1999;106:2269-80.

42. Wong TY, Klein R, Klein BE, Tielsch JM, Hubbard L, Nieto FJ. Retinal microvascular abnormalities and their relationship with hypertension, cardiovascular disease, and mortality. Surv Ophthalmol. 2001;46(1):59-80. 
43. Knudtson MD, Lee KE, Hubbard LD, Wong TY, Klein R, Klein BE. Revised formulas for summarizing retinal vessel diameters. Curr Eye Res. 2003; 27(3):143-9.

44. Prabhakar $\mathrm{P}$, Zhang $\mathrm{H}$, Chen $\mathrm{D}$, Faber JE. Genetic variation in retinal vascular patterning predicts variation in pial collateral extent and stroke severity. Angiogenesis. 2015;18(1):97-114.

45. Harris PA, Taylor R, Thielke R, Payne J, Gonzalez N, Conde JG. Research electronic data capture (REDCap) - A metadata-driven methodology and workflow process for providing translational research informatics support. J Biomed Inform. 2009;42(2):377-81.

46. Donald AE, Halcox JP, Charakida M, Storry C, Wallace SM, Cole TJ, et al. Methodological approaches to optimize reproducibility and power in clinical studies of flow-mediated dilation. J Am Coll Cardiol. 2008;51(20):1959-64.

47. Hsue PY, Lo JC, Franklin A, Bolger AF, Martin JN, Deeks SG, et al. Progression of atherosclerosis as assessed by carotid intima-media thickness in patients with HIV infection. Circulation. 2004;109(13):1603-8.

48. Jong E, Louw S, van Gorp EC, Meijers JC, ten Cate H, Jacobson BF. The effect of initiating combined antiretroviral therapy on endothelial cell activation and coagulation markers in South African HIV-infected individuals. Thromb Haemost. 2010;104(6):1228-34.

\section{Submit your next manuscript to BioMed Central and we will help you at every step:}

- We accept pre-submission inquiries

- Our selector tool helps you to find the most relevant journal

- We provide round the clock customer support

- Convenient online submission

- Thorough peer review

- Inclusion in PubMed and all major indexing services

- Maximum visibility for your research

Submit your manuscript at www.biomedcentral.com/submit 